\title{
RESEARCH ANNOUNCEMENTS THE SERIAL TEST FOR LINEAR CONGRUENTIAL PSEUDO-RANDOM NUMBERS
}

\author{
BY HARALD NIEDERREITER ${ }^{1}$ \\ Communicated by Jack Schwartz, August 22, 1977
}

Let $m \geqslant 2$ and $r$ be integers, let $y_{0}$ be an integer in the least residue system $\bmod m$, and let $\lambda$ be an integer coprime to $m$ with $\lambda \not \equiv \pm 1(\bmod m)$ and $(\lambda-1) y_{0}+r \not \equiv 0(\bmod m)$. A sequence $y_{0}, y_{1}, \ldots$ of integers in the least residue system $\bmod m$ is generated by the recursion $y_{n+1} \equiv \lambda y_{n}+r(\bmod m)$ for $n=0,1, \ldots$. In the homogeneous case $r \equiv 0(\bmod m)$, one chooses $y_{0}$ to be coprime to $m$. The sequence $x_{0}, x_{1}, \ldots$ in the interval $[0,1)$, defined by $x_{n}=y_{n} / m$ for $n=0,1, \ldots$, is a sequence of linear congruential pseudorandom numbers. The sequence is purely periodic; let $\tau$ denote its least period. In practice, $m$ is taken to be a large prime or a large power of 2 .

For a given $s \geqslant 2$, the serial test is set up to determine the amount of statistical dependence among $s$ successive terms in the sequence $x_{0}, x_{1}, \ldots$. To this end, one considers the s-tuples $x_{n}=\left(x_{n}, x_{n+1}, \ldots, x_{n+s-1}\right), n=0$, $1, \ldots$, and measures the deviation between the empirical distribution of the first $N$ of these $s$-tuples and the uniform distribution on $[0,1]^{s}$ by the quantity $D_{N}$ introduced in [3], where $1 \leqslant N \leqslant \tau$. For the homogeneous case, effective estimates for $D_{\tau}$ were established in [3], [4]. By extending techniques from [2] and [4], we can now handle the general case. Estimates for $D_{N}$ with $N<$ $\tau$ are of great practical interest because in calculations involving linear congruential pseudo-random numbers one only uses an initial segment of the period and not the full period itself.

The number $R^{(s)}(\lambda, m, q)$ is defined as in [3]. $C_{s}$ will denote an explicitly known constant depending only on $s$, whose exact value may be different in each occurrence.

THEOREM 1. For a prime $m$ we have

$$
D_{N}< \begin{cases}\frac{s}{m}+\frac{C_{s}}{\tau}(m-\tau)^{1 / 2}(\log m)^{s}+\frac{1}{2} R^{(s)}(\lambda, m, m) & \text { for } N=\tau, \\ \frac{s}{m}+\frac{C_{s}}{N} m^{1 / 2}(\log m)^{s+1}+\frac{1}{2} R^{(s)}(\lambda, m, m) & \text { for } 1 \leqslant N \leqslant \tau .\end{cases}
$$

AMS (MOS) subject classifications (1970). Primary 65C10; Secondary 10K05, 68A55.

${ }^{1}$ Supported by NSF Grant MCS 77-01699. 
Now let $m$ be a prime power, say $m=p^{\alpha}$ with $p$ prime and $\alpha \geqslant 2$. For $h \geqslant 1$, let $\mu\left(p^{h}\right)$ be the exponent to which $\lambda$ belongs $\bmod p^{h}$. Define a positive integer $\beta$ as follows: if $p$ is odd, let $\beta$ be the largest integer such that $p^{\beta}$ divides $\lambda^{\mu(p)}-1$; if $p=2$, let $\beta$ be the largest integer such that $2^{\beta}$ divides $\lambda^{\mu(4)}-1$. Furthermore, let $\kappa$ be the largest integer such that $p^{\kappa}$ divides $\lambda-1$, let $\omega$ be the largest integer such that $p^{\omega}$ divides $(\lambda-1) y_{0}+r$, and set $\gamma=\beta+\omega-\kappa$.

THEOREM 2. For a prime power $m=p^{\alpha}$, p prime, $\alpha \geqslant 2$, and $a \lambda$ with $\gamma<\alpha$ we have

$$
D_{N}<\left\{\begin{array}{l}
\frac{s}{m}+\frac{1}{2} R^{(s)}\left(\lambda, m, p^{\alpha-\gamma}\right) \quad \text { for } N=\tau, \\
\frac{s}{m}+\frac{C_{s}}{N}\left(\frac{m \tau}{\mu(m)}\right)^{1 / 2}(\log m)^{s+1}+\frac{1}{2} R^{(s)}\left(\lambda, m, p^{\alpha-\gamma}\right) \quad \text { for } 1 \leqslant N \leqslant \tau .
\end{array}\right.
$$

We note that in the frequently used special case $m=2^{\alpha}, \lambda \equiv 5(\bmod 8)$, and $r$ odd we have $\gamma=0$. The interpretation of these results is similar to that in $[3],[4]$.

The quantity $\rho^{(s)}(\lambda, m)$ introduced in [3] is convenient for computational purposes. Because of the above results and [3, Theorem 4], the reciprocal of $\rho^{(s)}(\lambda, m)$ may be taken as a measure for the amount of statistical dependence among $s$ successive terms in a sequence $x_{0}, x_{1}, \ldots$ having a large period $\tau$. The fact that this is really the correct indicator is shown by the following result.

THEOREM 3. For any $m, \lambda$, and $N$ with $1 \leqslant N \leqslant \tau$ we have

$$
D_{N} \geqslant \begin{cases}1 / s^{s} \rho^{(s)}(\lambda, m) & \text { for } 2 \leqslant s \leqslant 6, \\ \pi / 2(2 \pi+1)^{s} \rho^{(s)}(\lambda, m) & \text { for } s \geqslant 7 .\end{cases}
$$

We remark that the estimates for $D_{N}$ given here yield effective error bounds for Monte Carlo integrations using the points $\mathbf{x}_{0}, \mathbf{x}_{1}, \ldots, \mathbf{x}_{N-1}$ as nodes. This follows from general inequalities for the integration error in terms of $D_{N}$ which can be found in [1, Chapter 2, §5].

\section{REFERENCES}

1. L. Kuipers and H. Niederreiter, Uniform distribution of sequences, Wiley, New York, 1974.

2. H. Niederreiter, On the distribution of pseudo-random numbers generated by the linear congruential method. III, Math. Comp. 30 (1976), 571-597.

3. Statistical independence of linear congruential pseudo-random numbers, Bull. Amer. Math. Soc. 82 (1976), 927-929.

4. Pseudo-random numbers and optimal coefficients, Advances in Math. 26 (1977), 99-181. 